\title{
Sensitivity to the initial state of interacting ultracold bosons in disordered lattices
}

\author{
Benoît Vermersch and Jean Claude Garreau \\ Laboratoire de Physique des Lasers, Atomes et Molécules, \\ Université Lille 1 Sciences et Technologies, CNRS; F-59655 Villeneuve d'Ascq Cedex, Franc屯*
}

\begin{abstract}
We study the dynamics of a nonlinear one-dimensional disordered system obtained by coupling the Anderson model with the Gross-Pitaevskii equation. An analytical model provides us with a single quantity globally characterizing the localization of the system. This quantity obeys a scaling law with respect to the width of the initial state, which can be used to characterize the dynamics independently of the initial state.
\end{abstract}

\section{INTRODUCTION}

The Anderson model, introduced about 50 years ago [1], is the simplest model describing the effects of disorder in a quantum system in a relatively realistic way. It has however been recognized rather early that the model probably relied on too strong simplifications to match the actual behavior of electrons in a crystal: The Anderson model is a one-particle model at zero temperature. Moreover, the difficulty of experimental investigations in condensed matter systems [2] prompted a search for other systems where such effects could be observed in more favorable conditions, leading to studies of the localization of electromagnetic waves [3, 4] and sound waves [5, 6], where particle interactions are obviously absent. A breakthrough has been recently realized by using lasercooled atoms [7 -12], a development inscribed in a more general trend of studying many-body systems with ultracold atoms in optical lattices [13].

One of the long-standing questions concerning the Anderson model is the effect of particle-particle interactions. It was conjectured that the electron-electron coulomb repulsion would suppress localization. Including such effects in the model, however, implies going from a "simple" one-particle picture to a very complex many-body description. The problem of interactions is very rich in disordered ultracold atomic systems: For such systems, the interaction strength can be driven from repulsive to attractive, via the so-called Feshbach resonances [14]. Moreover, by choosing the atomic species, the quantum statistics can also be changed from Fermi-Dirac to Bose-Einstein (see, e.g., [15] and references therein). One can conjecture, for example, that attractive interactions shall increase localization in a cold Bose gas, but not in a Fermi gas. All possible combinations of the sign of interactions and quantum statistics can be similarly considered [16]. Mean-field theories, which simplify considerably the many-body problem, were generally considered to give a rather poor description of the electronic manybody problem in a crystal [17]. However, it became clear in recent years that these theories give, on the contrary, a very satisfactory description of ultracold Bose gases with

*URL: http://www.phlam.univ-lille1.fr/atfr/cq (weak) interactions in a variety of situations [13]. This puts into evidence the interest of studying cold atoms in the presence of disorder.

The first experimental result in this new field was the observation of "dynamical localization", a manifestation of the Anderson localization in the momentum space, by Raizen and co-workers, in 1994 [18]. Anderson localization of bosons in 1D [8] and 1D localization in the AubryAndré model [9], have also been observed experimentally, and, recently, observations of the 3D localization of fermions [10] and bosons [11] were claimed. The effect of interactions is also being studied experimentally [19 21]. Finally, the Anderson metal-insulator transition is being actively studied with a cold atom "quantum simulator" of the 3D Anderson model [22], including, the experimental determination of its critical exponent [7, 23, 24] and the study of its critical state [25].

\section{FRAME AND SCOPE}

We start by considering the tight-binding description of an one-dimensional (ordered) lattice of period $d$ obtained by projecting the eigenstates of the spatially periodic Hamiltonian on a basis of localized functions, usually Wannier functions $w_{n}(x)$, associated with each site $n$ :

$$
\psi_{\varepsilon}(x)=\sum_{n} c_{n} w_{n}(x)
$$

which produces a discretized eigenvalue problem

$$
V_{n} c_{n}+\sum_{r \neq 0} T_{r} c_{n+r}=\varepsilon c_{n}
$$

We use here the (usual) symmetric first-neighbors approximation $T_{r}=-T \delta_{r, \pm 1}$ and, as usual, rescale time such that $t \rightarrow \hbar t / T$ (and energies correspondingly: $E=$ $\varepsilon / T)$ in order to obtain the tight-binding equation:

$$
v_{n} c_{n}-c_{n-1}-c_{n+1}=E c_{n} \text {. }
$$

If all sites have the same diagonal energy, one can redefine the energy origin so that $v_{n} \equiv 0$, then [26, 27]:

$$
\begin{aligned}
E(q) & =-2 \cos q \\
\rho(q) & =1 / \pi
\end{aligned}
$$


where $q$ is the quasimomentum, $E(q)$ are the energies on the first band, $\rho$ is the density of states. The eigenfunctions $\psi_{q}(x)$ are delocalized Bloch waves of quasimomentum $q \in[0, \pi]$.

The Anderson model postulates that the main effect of crystalline disorder is to randomize the $v_{n}$ in eq. (2) in an interval $[-W / 2, W / 2]$, an approximation known as "diagonal disorder". The introduction of disorder redistributes the eigenenergies between the values $-2-W / 2$ and $2+W / 2$ and, more importantly, produces a localization of the corresponding eigenfunctions [43]. We shall index the eigenfunctions by $\nu=q-\pi / 2$ so that $\nu=0$ is at the center $q=\pi / 2$ of the energy band. The shape of a given eigenfunction depends on $E_{\nu}$ and on the realization $\left\{v_{n}\right\}$ of the disorder, but if one averages the eigenfunctions in a given energy interval $[E, E+\Delta E]$ over many realizations of the disorder one finds an exponential shape

$$
\overline{\psi_{\nu}}(n) \sim \exp \left(-\frac{\left|n-n_{\nu}\right|}{\ell_{\nu}(W)}\right)
$$

(we use overbars to indicate averages over realizations of the disorder) where $\ell_{\nu}(W)$ is the localization length. In the 1D case and in the limit of weak disorder one can show that [26]

$$
\ell_{\nu}(W) \approx \frac{96}{W^{2}} \cos ^{2} \nu,
$$

which vanishes at the borders of the band and has its maximum value $\ell_{0} \sim 96 \mathrm{~W}^{-2}$ at the center of the band. This localization can be interpreted by noting that, in the absence of disorder, all sites have identical energies, so the particle can tunnel from a site to the next one, generating a diffusive motion which leads, asymptotically, to a complete delocalization in the lattice. In the presence of diagonal disorder, however, it is unlikely that neighbor sites have close enough energies, so that in general, the particle can only perform virtual transition to neighbor sites, which leads to a localized exponential spreading of the eigenfunction.

We take into account interactions by using a meanfield approximation. The decomposition eq. (11) is used to transform [44] the Gross-Pitaevskii equation (or nonlinear Schrödinger equation) in a discrete nonlinear set of equations

$$
i \dot{c}_{n}=v_{n} c_{n}-c_{n-1}-c_{n+1}+g\left|c_{n}\right|^{2} c_{n}
$$

where $g$ is the parameter characterizing atom-atom interactions, proportional to the $s$-wave diffusion length [28, 29]. Disorder is introduced by randomizing $v_{n}$, as before. This model is occasionally named DANSE (Discrete Anderson Nonlinear Schrödinger Equation). As we use mean-field theory throughout this work, we shall use the terms "interaction" and "nonlinearity" interchangeably.

The main question in the problem of interacting disordered systems is, will interactions destroy localization? The DANSE model has been used, e.g. by Pikovskii and
Shepelyansky [30], to investigate this question. They observed numerically a revival of diffusion, leading, at very long times $\left(\sim 10^{7}\right)$, to a $\left\langle x^{2}\right\rangle \sim t^{\alpha}$ sub-diffusive behavior with $\alpha \approx 0.3$. Other works also tackled this problem, with somewhat contradictory results. For example, in [31], the use of the so-called "participation number" $\left(\sum_{n}\left|c_{n}\right|^{4}\right)^{-1}$ (instead of $\left.\left\langle x^{2}\right\rangle\right)$ as the quantity characterizing diffusion, lead to the conclusion that localization survives even in presence of a moderate nonlinearity, but subsequent studies [32, 33] confirmed the existence of a subdiffusive dynamics, with an exponent $\alpha$ that typically depends on the initial state. Experimental evidence of subdiffusion in the Aubry-André model has also been observed recently [21].

One can formally write the "diagona"l part of eq. (6) as $\left(v_{n}+g\left|c_{n}\right|^{2}\right) c_{n}$, which allows us to interpret the nonlinear term $v_{n}^{N L} \equiv g\left|c_{n}\right|^{2}$ as a "correction" (depending on the site population) to the energy of site $n$ [45]. This heuristic picture, although not rigorous, is often useful in interpreting the behavior of the system.

Mathematically, much is known about eq. (6) in the absence of disorder, in different contexts (see e.g. 34]): As a special case of the Ginsburg-Landau equation [35] it can describe some aspects of superfluidity, and, in optics, it describes the Kerr effect in a multimode system [36]. Two nonlinear effects play a particularly important role in our study. The first one is the called self-trapping, which manifests itself when a given site $n$ has a much larger population than its neighbors. In such case, the correction $v_{n}^{N L}$ decouples it from its neighbors (much as the disorder itself does), thus inhibiting diffusion. The second effect is the existence of chaotic behaviors (in a classical sense - that is, chaos related to sensitivity to initial conditions), due to the presence of nonlinearity $37-$ 39]. The chaotic evolution of the amplitudes $c_{n}$ may generate strong variations of the nonlinear correction $v_{n}^{N L}$, eventually bringing, even if only for a short time, neighbor sites close to degeneracy, thus favoring diffusion. In the presence of both disorder and interactions, different regimes are possible: Localization is expected to survive (or at least be destroyed only at very long times) if interactions are weak enough compared to disorder, diffusion (or subdiffusion) induced by the chaotic evolution is expected if these two effects are of the same order of magnitude, and self-trapping is expected to dominate, inhibiting diffusion, in the regime of strong interactions. These regimes have indeed been observed numerically [32, 33]. It can be tempting to classify these regimes by comparing $v_{n}^{N L}$ to $W$, but one must not forget that, as $v_{n}^{N L}$ depends on $c_{n}(t)$, the dynamics depends on the particular trajectory of the system in the $c_{n}$ space. Such a classification scheme is thus useless to define "phases" of the system.

This is an important and - from the point of view of the familiar linear quantum systems - unusual characteristic of nonlinear systems: The initial state plays a very important role (much more than in linear systems) in determining the dynamics. For example, if one takes, as in 

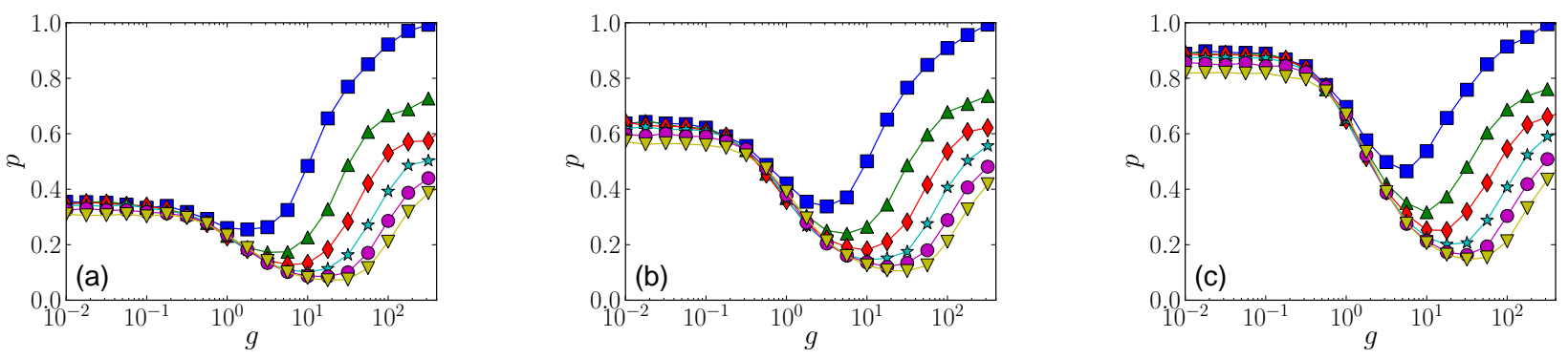

Figure 1: Survival probability $p\left(g, t=10^{5}\right)$ as a function of the nonlinear parameter $g$ for different values of the width $L_{0}$ of the initial state and disorder (a) $W=2$, (b) $W=3$, (c) $W=4$. Three regimes can be identified: Quasi-localized for low $g$; chaotic evolution with destruction of the localization for intermediate values of $g$; and self-trapping for high $g$. The nonlinearity destroys the localization almost completely in the chaotic regime, and self-trapping is more efficient, as expected, for smaller values of the initial state width $L_{0}$. For low disorder and small initial state width the localization due to self-trapping becomes much more efficient than the Anderson localization. Results are averaged typically over 1000 realizations of the disorder and of the initial phase distribution. Values of $L_{0}: 3$ (blue squares), 7 (green triangles), 13 (red diamonds), 21 (cyan stars), 31 (magenta circles), 41 (yellow inverted triangles).
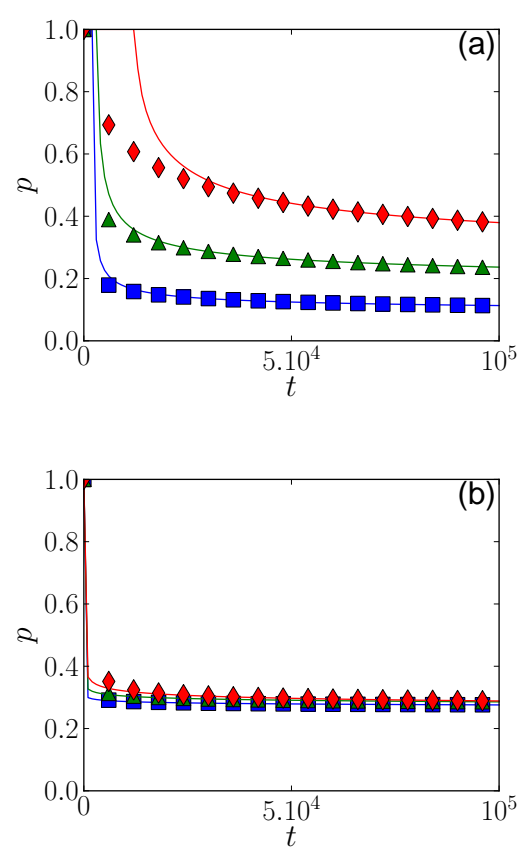

Figure 2: Comparison between numerical simulations (symbols) of $p(g, t)$ and the fit (solid lines) by eq. (8) for $L_{0}=31$ and (a) $g=1$, (b) $g=100$. Values of $W: 1$ (blue squares), 2 (green triangles) and 3 (red diamonds).

[30], an initial condition $c_{n}(t=0)=\delta_{n 0}$, one strongly favors self-trapping, and thus reduced diffusion. A choice like $c_{n}=1 / \sqrt{N}$ for $|n|<N / 2$ favors, for moderate values of $N$, chaotic dynamics, and thus diffusion. The main goal of the present work is to study the impact of initial conditions in the dynamics of the DANSE model. We shall show that this generally forbids a general classification of the dynamics in the parameter plane $g, W$. In the frame of a simplified model, introduced in sec. III, we shall however show that one can define an "effective wavepacket length" obeying scaling laws with respect to the extent of the initial state (sec. IV]). This allows us to define a generalized nonlinearity parameter, depending on the initial state, which leads to a more satisfactory classification of the dynamical behavior in the weak disorder limit. Finally, we discuss in sec. $\mathrm{V}$ the impact of some important neglected effects on our results.

\section{THE MODEL}

The search for asymptotic behaviors of the type $\left\langle x^{2}\right\rangle \sim$ $t^{\alpha}$ in the DANSE model implies describing the system by an array whose size increases with the evolution, the number of elements typically increasing as $t^{\alpha / 2}$. This is not our aim here: If one starts with an initial state localized in a relatively small region, the restoration of (sub-)diffusive dynamics by the nonlinearity should be apparent on the fact that parts of the wavepacket must continuously "escape" the initial region. We thus study a disordered lattice in a box $\mathrm{L}$ containing $L \sim 100$ sites, starting from an initial state of width $L_{0}$. At the frontiers $x= \pm L / 2$ of this box we place "absorbers" by adding an imaginary part $-i \eta_{a}$ to the potential, which increases exponentially over a distance $L_{a} \sim 10$, in order to prevent reflection of the parts of the wavepacket approaching the limits of the box.

We are interested here in the effect of the spatial extension of the initial wavepacket. In order to simplify the problem, we use a initial wavepacket of square shape and width $L_{0}$ :

$$
\left|c_{n}(0)\right|= \begin{cases}\left(L_{0}\right)^{-1 / 2} & |n| \leq\left(L_{0}-1\right) / 2 \\ 0 & \text { otherwise. }\end{cases}
$$

We prevent dominant quantum interference effects by set- 

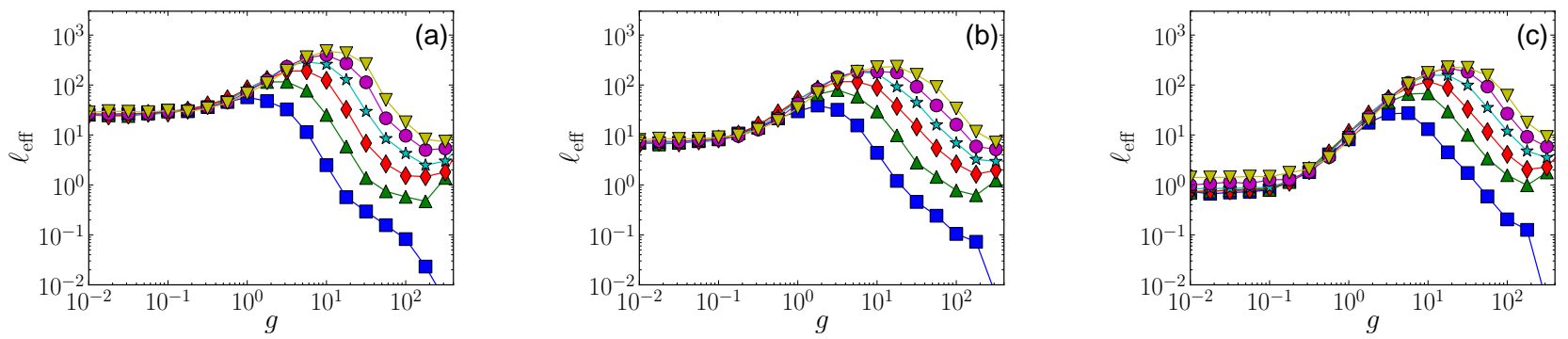

Figure 3: Effective localization length as a function of the nonlinearity $g$, for (a) $W=2$, (b) $W=3$ and (c) $W=4$ and various values of the width $L_{0}$ of the initial state. Note that for $g \rightarrow 0$ we retrieve the linear localization length $\ell_{0} \approx 96 W^{-2}$ in the weak disorder limit $W \leq 3$. Same graphic conventions as in fig. 1 .
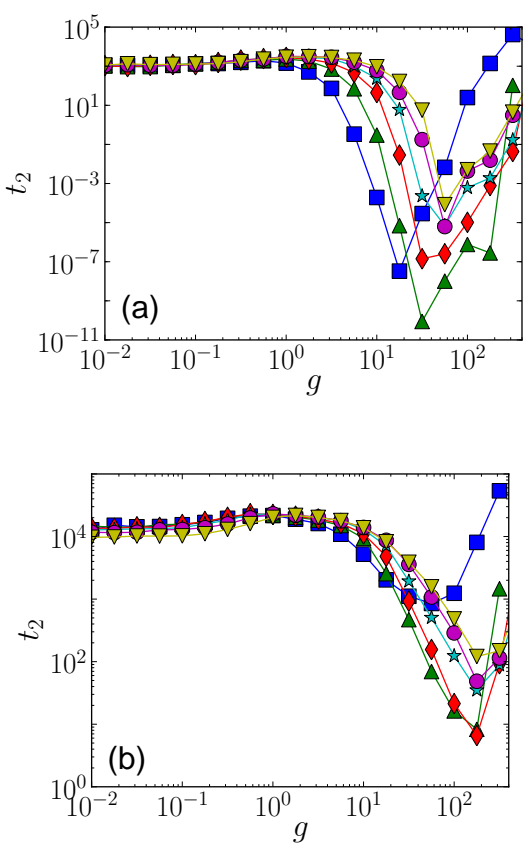

Figure 4: Activation time $t_{2}$ as a function of the nonlinearity $g$ for (a) $W=2$ and (b) $W=4$ and various values of the width $L_{0}$ of the initial state. Same graphic conventions as fig. 1 .

ting random phases to the $c_{n}$. We shall consider in sec. $V$ the effect of non-random quantum phases.

The part of the wavepacket that, at time $t$, has remained in the box L (that we shall call the "survival probability") is given by

$$
p(g, t)=\sum_{n=-(L-1) / 2}^{(L-1) / 2}\left|c_{n}(t)\right|^{2} .
$$

We display in fig. $11 p(g, t)$ calculated at $t=10^{5}$ as a function of the nonlinearity $g$ for various sizes of the initial state $L_{0}$, for a disorder amplitude $W=2$ (part (a) of fig. 11), $W=3$ (b) and $W=4$ (c). The numerical integration is done by the Crank-Nicholson method [40]. The probability density $\left|c_{n}\right|^{2}$ is averaged over typically 1000 realizations of disorder $\left\{v_{n}\right\}$ and of the initial phases $\arg \left[c_{n}(0)\right]$. Note that one has $p\left(g \rightarrow 0, t=10^{5}\right)<1$, due to the fact that the initial state typically projects onto an ensemble of localized eigenstates with localization lengths given by eq. (5). Some of these eigenstates have localization lengths large enough to "touch" the borders of the box L, so that the projection itself leads to losses. One can see by comparing the three plots of fig. 1 that these losses decrease with the disorder $W$, and are independent of $L_{0}$, as expected for the linear regime.

From fig. 1 one can identify three main dynamical regimes for increasing nonlinearity parameter $g$ : i) A "quasi-localized" dynamical regime in which localization is only weakly perturbed by the nonlinearity; ii) a dynamical regime in which localization is destroyed by the chaotic dynamics [46], the diffusion is reestablished and the losses increase notably; iii) self-trapping regime in which diffusion (thus losses) is inhibited again. It is interesting to note that, for concentrated initial states $L_{0} \lesssim 20$ and weak disorder, self-trapping can be much more efficient than localization in suppressing diffusion. These dynamical regimes correspond to those studied e.g. in Laptyeva et al.. 33] by considering the long-term behavior of $\left\langle x^{2}\right\rangle$. Fig. 1 1 shows that the crossover between these regimes strongly depends on the width of the initial state.

In the absence of nonlinearity, the initial state evolves until it takes an exponentially localized shape of width $\sim \ell_{0}$ and then "freezes". In the presence of the nonlinearity, one observes a global enlarging of the wavefunction, which results in a increasing absorption at the borders of the box L. In the weak disorder limit we can construct an analytic model for these losses, starting from the properties of the linear $(g=0)$ system. This model, discussed in appendix A, produces an analytical expression describing the asymptotic behavior of the survival probability $p(g=0, t)$, eq. A5 . Remarkably, a small modification of this expression furnishes also a function describing the asymptotic behavior in the nonlinear case, for values of $g$ as large as $10^{3}$. This expression is 

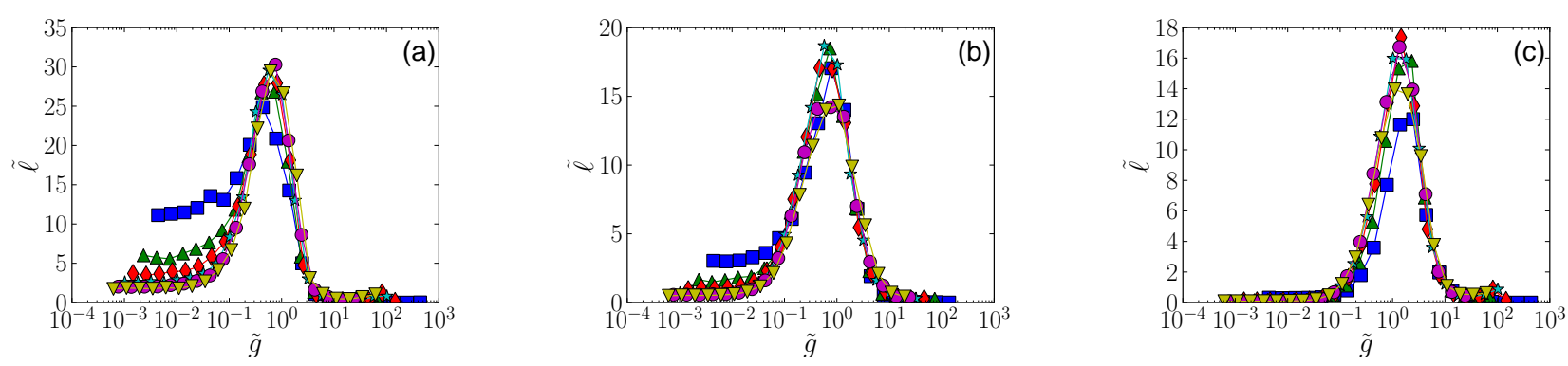

Figure 5: Scaling law. The same data of fig. 3 is plotted in terms of the scaled quantities $\widetilde{g}=g L_{0}^{-3 / 4}$ and $\widetilde{\ell}=\ell_{\mathrm{eff}} L_{0}^{-3 / 4}$ for (a) $W=2$, (b) $W=3$ and (c) $W=4$. A clear grouping of the curves in the nonlinear region is observed. Same graphic conventions as fig. 1 .
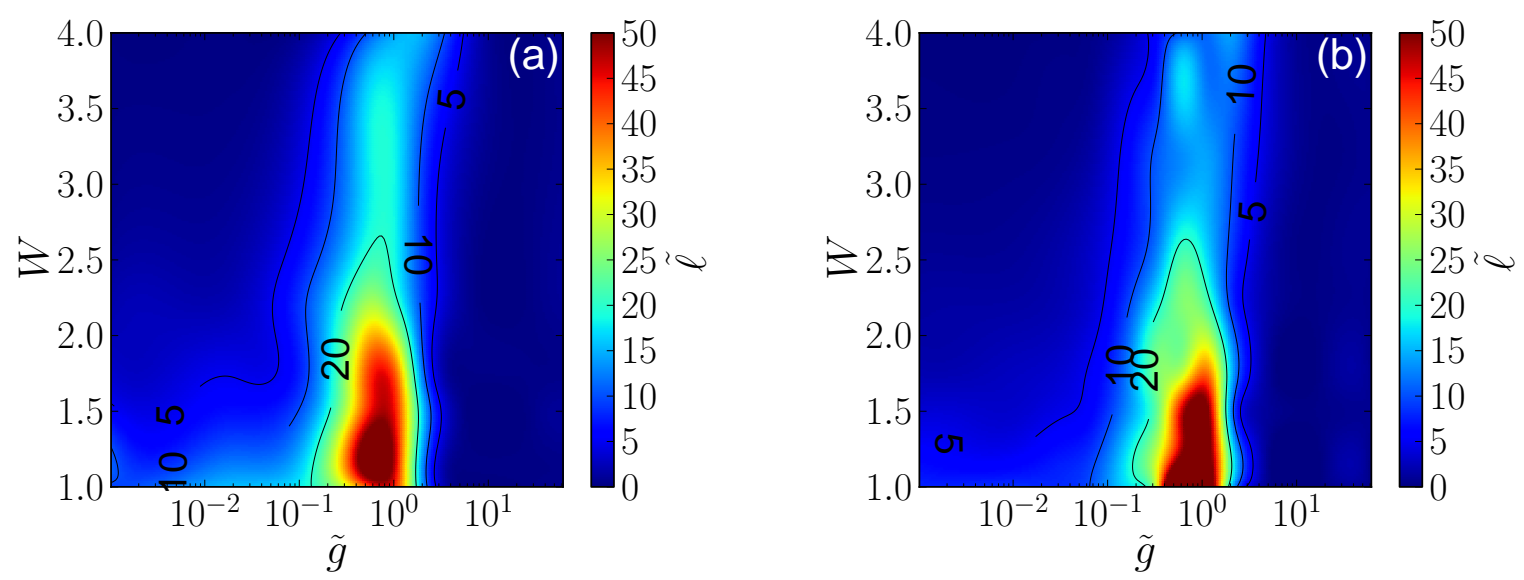

Figure 6: False colors plot of the scaled effective wavepacket length $\tilde{\ell}$ as a function of the scaled nonlinearity $\tilde{g}$ and of the disorder $W$ for (a) $L_{0}=21$ and (b) $L_{0}=41$. The structure of the parameter space is very similar for the two values of the initial state width.

$$
p(g, t)=\frac{2}{\pi} \sin ^{-1}\left(\sqrt{\frac{2 \ell_{a}}{\ell_{\mathrm{eff}} \log \left(t / t_{2}\right)+2 \ell_{a}}}\right) \quad\left(t \gg t_{2}\right),
$$

where $\ell_{a}$ is given by eq. (A6), depending on the geometry and on the localization length $\ell_{0}(W)$, and $\ell_{\text {eff }}$ and $t_{2}$ fitting parameters. With respect to eq. A5 of app. A we simply replaced $\ell_{0}$ by an "effective wavepacket length" $\ell_{\text {eff }}$ which is the second fitting parameter of our model. Fig. 2 shows that the asymptotic behavior of the survival probability is very well fitted by this formula. The quantity $\ell_{\text {eff }}$ plays a major role in the present work, as it can globally characterize the wavepacket shape. We attribute this somewhat astonishing property of our model to the fact that, even in presence of the nonlinearity, the wavepacket displays exponential wings in the asymptotic regime, as in can be verified numerically.

We display in fig. 3 the behavior of the effective wavepacket length $\ell_{\text {eff }}$ as a function of the nonlinear- ity, for disorder parameters $W=1$ [plot (a)], $W=2$ [plot (b)], and $W=3$ [plot (c)] and for various values of the initial state width $L_{0}$. One clearly identifies the regions corresponding to the three dynamical regimes discussed above: Quasi-localized regime, characterized by a constant value of $\ell_{\text {eff }}$, independent of $g$ and $L_{0}$; chaotic regime, characterized by a marked increasing of $\ell_{\text {eff }}$ with $g$ and strongly dependent on $L_{0}$; and self-trapping regime, in which $\ell_{\text {eff }}$ decreases again and can even become smaller than the Anderson localization length $\ell_{0}$, and which is also dependent on $L_{0}$. Fig. 4 shows the behavior of the "activation time" $t_{2}$ (see App. A for the same set of states and parameters as fig. 3. This time roughly corresponds the time for the wavepacket to "touch" the border of the box L, when 
losses become important. In the quasilocalized regime $t_{2}$ is thus very high. The onset of the chaotic behavior, favoring diffusion, produces a dramatic decrease of $t_{2}$ which becomes virtually zero (that is, losses began almost instantaneously). Self-trapping, inhibiting diffusion, produces an increase of $t_{2}$. The same three dynamical regimes can hence be observed also in the behavior of $t_{2}$. This fit parameter, although necessary to obtain a good agreement with numerical simulation, thus carries essentially the same information as $\ell_{\text {eff }}$. For $g \rightarrow \infty$, one expects the wave packet to evolve very little due to "immediate" self-trapping, that is, its asymptotic width should be proportional $L_{0}$. In fig. 3, $\ell_{\text {eff }}$ clearly has not attained this large $g$ regime, but we observed numerically that even for moderate values of $g \sim 300$ it is roughly proportional to $L_{0}: \ell_{\mathrm{eff}}(g=300) \approx 0.25 L_{0}$ for $W$ ranging from 2 to 4 .

\section{SCALING LAWS}

The curves in fig. 3 suggest the existence of scaling laws. In fig. [5 we show a plot of the quantity $\widetilde{\ell} \equiv \ell_{\text {eff }} L_{0}^{-3 / 4}$ as a function of the scaled nonlinearity defined by

$$
\widetilde{g} \equiv g L_{0}^{-3 / 4}
$$

using the same data of fig. 3. One observes, at least for the $\widetilde{g}>1$, a clear grouping of the curves, which indicates that the scaling applies essentially to the nonlinear part of the behavior, as intuitively expected: Indeed, as $\ell_{\mathrm{eff}}(g=0) \rightarrow \ell_{0}, \tilde{\ell}(g=0) \sim 96 W^{-2} L_{0}^{-3 / 4}$ which is dependent of $L_{0}$; this fact simply means that Anderson localization is not controlled by $g$. On the nonlinearitydominated region, however, one would (naively) expect the nonlinear effects to scale as $\left\langle v_{n}^{N L}\right\rangle \sim g L_{0}^{-1}$ for an initial state uniformly populating $L_{0}$ states. Presently, we have no convincing explanation for the additional $L_{0}^{1 / 4}$ factor. We note that the scaling is not perfect for the small values of $L_{0}$, for which the self-trapping is effective even for low values of $g$.

Fig. 5 shows that the scaling allows us to define crossovers between the three dynamical regimes which $d o$ not depend on $L_{0}$. This is confirmed in fig. 6, in which we plotted in false-colors the scaled effective wavepacket length $\tilde{\ell}$ in the parameter plane $\tilde{g}, W$ for $L_{0}=21$ [plot (a)] and for $L_{0}=41$ [plot (b)]. Despite of the factor 2 in the width of the initial state, the two plots are almost identical. The crossover between the quasi-localized regime and the chaotic regime is found to be around $\tilde{g}_{c} \approx 0.1$ and the crossover between the chaotic regime and the self-trapping regime around $\tilde{g}_{s t} \approx 5$.

The use of these scaled variables thus allows us to characterize the nonlinear dynamics independently of the size of the initial state, which constitutes an important step in the understanding of these complex dynamics.

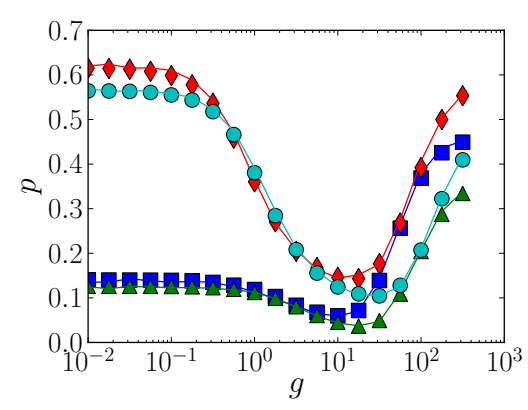

Figure 7: Survival probability at $t=10^{5}$ for a square initial state (full lines) and a gaussian initial state eq. (10) (symbols), for $W=1, L_{0}=21$ (blue/squares), $W=1, L_{0}=41$ (green/triangles), $W=3, L_{0}=21$ (red/diamonds) and $W=3, L_{0}=41$ (cyan/circles). The dependence on the shape of the initial state is very small.

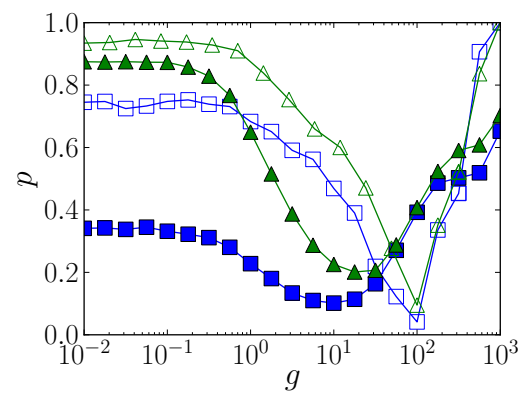

Figure 8: Survival probability at $t=10^{5}$ for states with coherent (empty symbols) and incoherent (full symbols) initial phases, for $W=2, L_{0}=21$ (blue squares) and $W=4, L_{0}=$ 21, (green triangles). The coherent case presents a marked enhancement on the transport for $g \approx 100$.

\section{OTHER EFFECTS}

In this section, we consider three potentially significant effects not taken into account above.

(i) The shape of the initial state. In Fig. 7, we compared the results discussed above, obtained with the "square" initial state defined by eq. (7) with results obtained with an initial gaussian state

$$
\left|c_{n}(0)\right|^{2}=\frac{1}{\sqrt{2 \pi} \sigma} e^{-n^{2} / 2 \sigma^{2}}
$$

We set $\sigma=\sqrt{\left(L_{0}-1\right)\left(L_{0}+1\right) / 12}$ so that, for a given $L_{0}$, both square and gaussian initial states have the same second momentum $\sum n^{2}\left|c_{n}\right|^{2}$. We see that the dynamics is independent of the shape of the initial state to a very good approximation. In the self-trapping regime, we see that the dynamics depends more on the size $L_{0}$ than on the shape of the initial state.

(ii) The effect of the quantum phases of the initial state. Our choice of using initial random (incoherent) phases in 


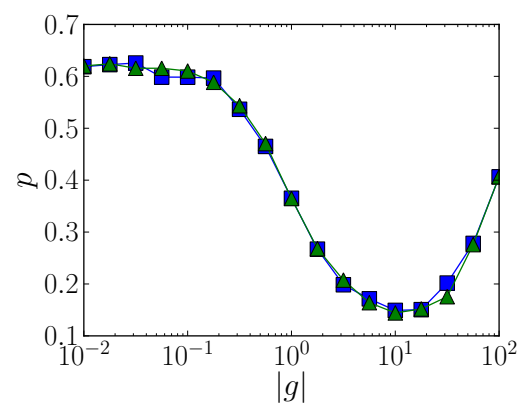

Figure 9: Effect of the sign of the nonlinearity with $L_{0}=21$ and $W=3$. The curves display the survival probability at $t=10^{5}$ for $g>0$ (blue squares) and $g<0$ (green triangles).

the initial state proved useful in allowing us to give a global characterization of the dynamics. However, a particular coherent combination of $c_{n}$ phases can generate effects of quantum interference with an impact on the dynamics. This is illustrated in Fig. 8, where we compared the dynamics of an initial state of random phases and an initial state with a constant phase for all $c_{n}$. The latter one presents a different behavior, with a marked increasing in the transport for $g \approx 100$, which means that chaotic behavior is highly favored in this case. Our previous result remains however a valid description of the "average" dynamics.

(iii) The effect of the sign of the nonlinearity. In all the results presented above $g>0$, that is, we have repulsive interactions. In the limits of our approach, we have seen no significant difference between attractive and repulsive interactions, see fig. 9] in contrast with some theoretical speculations. This confirms and generalizes a result obtained in ref. [41] in a different context.

Despite the simplifications we made in order to make this problem tractable, it appears that our conclusions represent very well the general behavior of the system, independently of most microscopic parameters. We can thus say that we have characterized the dynamics in a rather universal way.

\section{CONCLUSION}

Nonlinear dynamics is, in general, highly sensitive to initial conditions, and no global characterization of the dynamics can be made unless one can correctly take into account this dependence. This is particularly true for the very important system studied in the present work: The mean-field generalization of the Anderson model, described by the Gross-Pitaevskii equation. We proposed a quantity providing a global characterization of the dynamics independent of the size of the initial state, the scaled effective wavepacket length $\tilde{\ell}$. This is a first step in the necessary development of a coherent language describing the effects of nonlinearities in Quantum Mechan- ics, which will, most probably, constitute one of the major subjects of atomic physics in the next years.

\section{Acknowledgments}

The authors are grateful to D. Delande and V. Zehnlé for fruitful discussions. Laboratoire de Physique des Lasers, Atomes et Molécules is UMR 8523 of CNRS. Work partially financed by the Agence Nationale de la Recherche (MICPAF and LAKRIDI grants).

\section{Appendix A: Determination of $p(g=0, t)$}

The aim of this appendix is to find an analytical formula describing the evolution of the survival probability in the linear case $g=0$. Let us first recall a few important results in the case of an infinite lattice (no losses - standard 1D Anderson model). In this case, the corresponding Anderson Hamiltonian $H_{A}$ admits eigenstates $|\nu\rangle$ of energy $E_{\nu}$. In real space representation, $\psi_{\nu}(n)=\langle n \mid \nu\rangle$ is centered at position $n_{\nu}$ in the lattice, with a localization length $\ell_{\nu} \leq \ell_{0}(W)$. As such states are exponentially localized in the average, the density of presence of these eigenstates at the site $n$ is

$$
|\langle n \mid \nu\rangle|^{2} \approx \tanh \left(1 / \ell_{\nu}\right) e^{-2\left|n-n_{\nu}\right| / \ell_{\nu}}
$$

In our problem we set an exponential imaginary potential (absorber) on the border sites of the box $V_{i}(n)=$ $-i \eta_{a} \exp \left(|n| / n_{c}\right) \quad(|n|>L / 2)$. In order to make the problem tractable, we make the assumption that the imaginary potential can be characterize by a constant value $V_{i}=-i \eta_{a}$. The corresponding Schrödinger equation is

$$
i \frac{\partial|\psi(t)\rangle}{\partial t}=H_{A}|\psi(t)\rangle+V_{i}|\psi(t)\rangle
$$

Decomposing the wave function in the eigenfunctions basis, $|\psi(t)\rangle=\sum_{\nu} \alpha_{\nu} e^{-i E_{\nu} t}|\nu\rangle$, we obtain (setting $\dot{\alpha}_{\nu} \equiv$ $\left.\partial \alpha_{\nu} / \partial t\right)$ :

$$
i \dot{\alpha}_{\mu}=\sum_{\nu, n}\langle\mu \mid n\rangle\langle n \mid \nu\rangle V_{i}(n) \alpha_{\nu} \exp \left[i\left(E_{\mu}-E_{\nu}\right) t\right] .
$$

In the average, the contribution of $\mu \neq \nu$ is negligible and, using eq. (A1), we get

$$
i \dot{\alpha}_{\nu}=\left(\tanh \left(1 / \ell_{\nu}\right) \sum_{n} V_{i}(n) \exp \left(-2\left|n-n_{\nu}\right| / \ell_{\nu}\right)\right) \alpha_{\nu}
$$

Without loss of generality, we assume $n_{\nu} \geq 0$ and note $\ell_{a}$ the distance between the center of the wave packet corresponding to this eigenstate and the closest border 


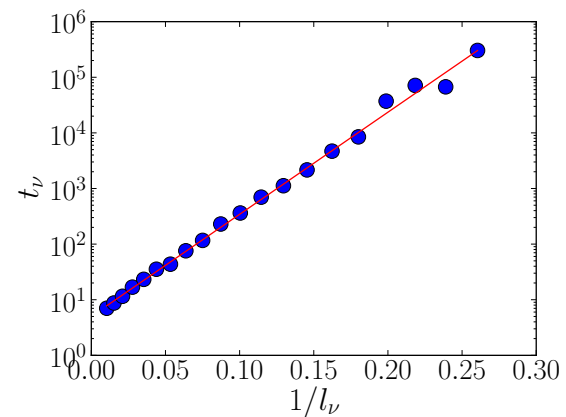

Figure 10: The blue circles correspond to the values of $t_{\nu}$ obtained by fitting the numerically calculated evolution of $\alpha_{\nu}$ with an exponential [eq. (A2)]. The red solid line is a fit of these points by eq. (A3).

of the box at $n=L / 2$. Then

$$
\begin{aligned}
\dot{\alpha}_{\nu} & =-\eta_{a}\left(\tanh \left(1 / \ell_{\nu}\right) \sum_{n \geq n_{\nu}+\ell_{a}} e^{-2\left(n-n_{\nu}\right) / \ell_{\nu}}\right) \alpha_{\nu} \\
& =-\frac{\alpha_{\nu} \eta_{a}}{1+e^{-2 / \ell_{\nu}}} e^{-2 \ell_{a} / \ell_{\nu}} .
\end{aligned}
$$

For moderate values of the disorder we can assume $1+$ $e^{-2 / \ell_{\nu}} \approx 1$, and obtain, integrating the above equation,

$$
\begin{aligned}
\alpha_{\nu} & =\alpha_{\nu}(0) e^{-t / 2 t_{\nu}} \\
t_{\nu} & =\frac{1}{2 \eta_{a}} e^{2 \ell_{a} / \ell_{\nu}} .
\end{aligned}
$$

We thus established two important results: (i) the attenuation (due to the absorber) of an eigenstate is exponential, and (ii) the typical time scale of absorption is proportional to $\exp \left(2 \ell_{a} / \ell_{\nu}\right)$ [47]. These results can be generalized for an imaginary potential that varies exponentially. We calculated numerically the evolution of $\alpha_{\nu}$ (with a non-constant imaginary potential) for a few eigenstates (averaged over the disorder) and fitted this evolution with equation (A2) to obtain the corresponding values of $t_{\nu}$, represented as circles in Fig. 10, plotted as a function of $1 / \ell_{\nu}$ in semi-log scale. The dependence of $t_{\nu}$ on $\ell_{\nu}$ can in turn be fitted with the relation (red line in Fig. 10)

$$
t_{\nu}=t_{2} \exp \left[2 \ell_{a}\left(1 / \ell_{\nu}-1 / \ell_{0}(W)\right)\right] .
$$

The fit parameter $t_{2}$ thus corresponds to the typical time-scale for the attenuation of the eigenstate of largest width.

The evolution of the survival probability $p$ can be calculated for a uniform initial state over $L_{0}$ Anderson states, i.e. $\left|\alpha_{\nu}(0)\right|^{2}=1 / L_{0}$. From eq. (A2)

$$
p(g=0, t)=\sum_{\nu}\left|\alpha_{\nu}\right|^{2}=\frac{1}{L_{0}} \sum_{\nu} \exp \left(-t / t_{\nu}\right)
$$

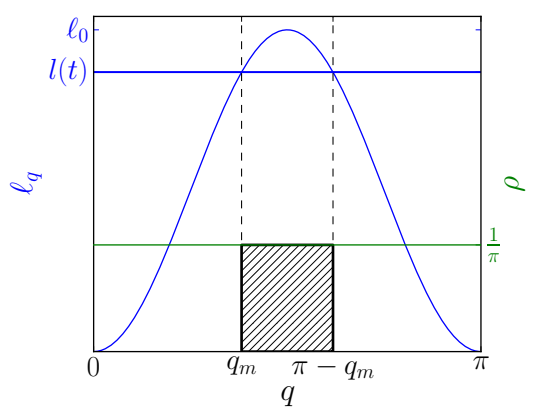

Figure 11: Graphical determination of $p$ in the weak-disorder limit. The curve represents $\ell_{0} \sin ^{2} q$ and its intersection with $\ell(t)$ determines $q_{m}$. The dashed region correspond to the integral eq. A4).

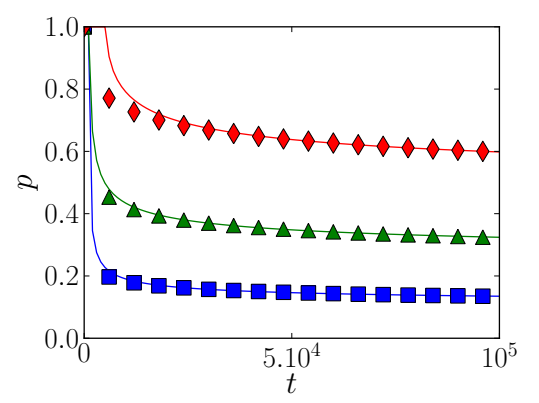

Figure 12: Comparison between eq. A5 and numerical simulations for $L_{0}=31$. The asymptotic behavior of the survival probability is very well described by our formula, for different values of the disorder, $W=1$ (blue squares), $W=2$ (green triangles), $W=3$ (red diamonds).

with $t_{\nu}$ is given by eq. (A3). We now take $t_{\nu}$ as the typical time for decay of the eigenstate $\nu$, i.e. we approximate $\exp \left(-t / t_{\nu}\right) \approx \Theta\left(t_{\nu}-t\right)$, where $\Theta$ is the Heaviside function:

$$
p(g=0, t)=\frac{1}{L_{0}} \sum_{\nu} \Theta\left(t_{\nu}-t\right) .
$$

That is, we consider that the eigenstate is completely absorbed at time $t_{\nu}$ at which it "touches" the absorbing potential. From eq. A3 we see that

$$
\ell_{\nu}=\frac{2 \ell_{a}}{\log \left(t_{\nu} / t_{2}\right)+2 \ell_{a} / \ell_{0}}
$$

At time $t$, the surviving states are thus those such that

$$
\ell_{\nu}<=\ell(t) \equiv \frac{2 \ell_{a}}{\log \left(t / t_{2}\right)+2 \ell_{a} / \ell_{0}} .
$$

In the weak-disorder limit one can use eq. (3) and write

$$
p(g=0, t)=\int_{\ell_{q}<\ell(t)} \rho d q,
$$


where $\rho=1 / \pi$ is the density of states [eq. (4)]. The condition $\ell_{q}<\ell(t)$ sets a maximum value $q_{m}$ of the quasimomentum that can be determined graphically, as shown in Fig. (11). The condition

$$
\ell(t)=\ell_{0} \sin ^{2} q_{m}
$$

gives

$$
q_{m}=\sin ^{-1}\left(\ell(t) / \ell_{0}\right)^{1 / 2}
$$

and, finally

$$
p(g=0, t)=\frac{2 q_{m}}{\pi}=\frac{2}{\pi} \sin ^{-1}\left(\frac{2 \ell_{a} / \ell_{0}}{\log \left(t / t_{2}\right)+2 \ell_{a} / \ell_{0}}\right)^{1 / 2}
$$

Fitting the numerical survival probability with the above expression, best results are obtained by setting

$$
\ell_{a}=\frac{1}{2}\left(\frac{L}{2}-\frac{L_{0}}{4}-\frac{\ell_{0}}{5}\right),
$$

as shown in Fig. 12, One can think that the distance $L / 2-L_{0} / 4$ is more likely to represent the typical distance from the initial state to the absorber. The factor $1 / 2$ probably compensates for the brutal step we made above of approximating the exponential function by the Heaviside function. The presence of the term in $\ell_{0}$ has a physical meaning: In our simulations, we initially excite $L_{0}$ Wannier states, not $L_{0}$ Anderson states and as a consequence, the initial size of the wave packet is larger than $L_{0}$.
[1] P. W. Anderson, Phys. Rev. 109, 1492 (1958).

[2] P. A. Lee and T. V. Ramakrishnan, Rev. Mod. Phys. 57, 287 (1985).

[3] D. S. Wiersma, P. Bartolini, A. Lagendijk, and R. Righini, Nature (London) 390, 671 (1997).

[4] M. Störzer, P. Gross, C. M. Aegerter, and G. Maret, Phys. Rev. Lett. 96, 063904 (2006).

[5] H. Hu, A. Strybulevych, J. H. Page, S. E. Skipetrov, and B. A. van Tiggelen, Nat. Phys. 4, 945 (2008).

[6] S. Faez, A. Strybulevych, J. H. Page, A. Lagendijk, and B. A. van Tiggelen, Phys. Rev. Lett. 103, 155703 (2009).

[7] J. Chabé, G. Lemarié, B. Grémaud, D. Delande, P. Szriftgiser, and J. C. Garreau, Phys. Rev. Lett. 101, 255702 (2008).

[8] J. Billy, V. Josse, Z. Zuo, A. Bernard, B. Hambrecht, P. Lugan, D. Clément, L. Sanchez-Palencia, P. Bouyer, and A. Aspect, Nature (London) 453, 891 (2008).

[9] G. Roati, C. d'Errico, L. Fallani, M. Fattori, C. Fort, M. Zaccanti, G. Modugno, M. Modugno, and M. Inguscio, Nature (London) 453, 895 (2008).

[10] S. S. Kondov, W. R. McGehee, J. J. Zirbel, and B. DeMarco, Science 334, 66 (2011).

[11] F. Jendrzejewski, A. Bernard, K. Mueller, P. Cheinet, V. Josse, M. Piraud, L. Pezzé, L. Sanchez-Palencia, A. Aspect, and P. Bouyer, Three-dimensional localization of ultracold atoms in an optical disordered potential, arXiv:1108.0137v2 [cond-mat.other] (2011).

[12] H. Lignier, J. Chabé, D. Delande, J. C. Garreau, and P. Szriftgiser, Phys. Rev. Lett. 95, 234101 (2005).

[13] I. Bloch, J. Dalibard, and W. Zwerger, Rev. Mod. Phys. 80, 885 (2008).

[14] C. Chin, R. Grimm, P. Julienne, and E. Tiesinga, Rev. Mod. Phys. 82, 1225 (2010).

[15] L. Sanchez-Palencia and M. Lewenstein, Nat. Phys. 6, 87 (2010).

[16] F. Crépin, G. Zaránd, and P. Simon, Phys. Rev. Lett. 105, 115301 (2010).

[17] A. L. Fetter and J. D. Walecka, Quantum Theory of Many-Particle Systems (Dover, New York, USA, 2003).

[18] F. L. Moore, J. C. Robinson, C. Bharucha, P. E. Williams, and M. G. Raizen, Phys. Rev. Lett. 73, 2974
(1994).

[19] B. Deissler, M. Zaccanti, G. Roati, C. d'Errico, M. Fattori, M. Modugno, G. Modugno, and M. Inguscio, Nat. Phys. 6, 354 (2010).

[20] B. Deissler, E. Lucioni, M. Modugno, G. Roati, L. Tanzi, M. Zaccanti, M. Inguscio, and A. G. Modugno, New J. Phys 13, 023020 (2011).

[21] E. Lucioni, B. Deissler, L. Tanzi, G. Roati, M. Zaccanti, M. Modugno, M. Larcher, F. Dalfovo, M. Inguscio, and G. Modugno, Phys. Rev. Lett. 106, 230403 (2011).

[22] G. Casati, I. Guarneri, and D. L. Shepelyansky, Phys. Rev. Lett. 62, 345 (1989).

[23] G. Lemarié, J. Chabé, P. Szriftgiser, J. C. Garreau, B. Grémaud, and D. Delande, Phys. Rev. A 80, 043626 (2009).

[24] M. Lopez, J. F. Clément, P. Szriftgiser, J. C. Garreau, and D. Delande, Experimental Test of Universality of the Anderson Transition, arXiv:1108.0630v1 [quant-ph] (2011).

[25] G. Lemarié, H. Lignier, D. Delande, P. Szriftgiser, and J. C. Garreau, Phys. Rev. Lett. 105, 090601 (2010).

[26] J. M. Luck, Systèmes désordonnés unidimensionnels (AlÃ@)a Sacaly, Gif sur Yvette, France, 1992).

[27] N. W. Ashcroft and N. D. Mermin, Solid State Physics (Saunders College Publishing, Fort Worth, USA, 1976).

[28] F. Dalfovo, S. Giorgini, L. Pitaevskii, and S. Stringari, Rev. Mod. Phys. 71, 463 (1999).

[29] C. J. Pethick and H. Simth, Bose-Einstein Condensation in Dilute Gases (Cambridge University Press, Cambridge, UK, 2008), 2nd ed.

[30] A. S. Pikovsky and D. L. Shepelyansky, Phys. Rev. Lett. 100, 094101 (2008).

[31] G. Kopidakis, S. Komineas, S. Flach, and S. Aubry, Phys. Rev. Lett. 100, 084103 (2008).

[32] S. Flach, D. O. Krimer, and C. Skokos, Phys. Rev. Lett. 102, 024101 (2009).

[33] T. V. Laptyeva, J. D. Bodyfelt, D. O. Krimer, C. Skokos, and S. Flach, EPL (Europhysics Letters) 91, 30001 (2010).

[34] C. Sulem and P. L. Sulem, The Nonlinear Schrödinger Equation (Springer-Verlag, Berlin, Germany, 1999). 
[35] J. F. Annett, Superconductivity, Superfluids, and Condensates (Oxford University Press, 2004).

[36] A. Yariv, Optical Electronics (Saunders College Publishing, Fort Worth, USA, 1991), 4th ed.

[37] Q. Thommen, J. C. Garreau, and V. Zehnlé, Phys. Rev. Lett. 91, 210405 (2003).

[38] G. P. Berman, F. Borgonovi, F. M. Izrailev, and A. Smerzi, Phys. Rev. Lett. 92, 030404 (2004).

[39] L. Fallani, L. de Sarlo, J. E. Lye, M. Modugno, R. Saers, C. Fort, and M. Inguscio, Phys. Rev. Lett. 93, 140406 (2004).

[40] W. H. Press, S. A. Teukolsky, T. Vetterling, and B. P. Flannery, Numerical Recipes in $C$ (Cambridge University Press, Cambridge, UK, 2007), 3rd ed.

[41] L. Rebuzzini, S. Wimberger, and R. Artuso, Phys. Rev.
E 71, 036220 (2005).

[42] G. Casati, G. Maspero, and D. L. Shepelyansky, Phys. Rev. Lett. 82, 524 (1999).

[43] In one dimension, all eigenfunctions are localized, whatever the disorder $W$.

[44] In order to do so one generalizes the first-neighbors approximation by supposing that $\int d x w_{n}^{3}(x) w_{n \pm 1}(x) \ll$ $\int d x w_{n}^{4}(x)$ and keeps only the "diagonal" nonlinear term.

[45] Note that $\left\langle v_{n}^{N L}\right\rangle \approx \mu$, where $\mu$ is the chemical potential.

[46] The study of the site population dynamics, which displays in such case continuous spectrum, confirms the presence of a chaotic behavior.

[47] A similar form has been found in ref. [42]. 